\title{
ANOTHER VIEW OF THE WEIERSTRASS THEOREM ${ }^{1}$
}

\author{
KENNETH JOHN PRESKENIS
}

\begin{abstract}
We present two theorems which conclude that polynomials in $z$ and a given continuous function $f$ are dense in all continuous complex valued functions on the closed unit disk. The first theorem requires that $f$ be differentiable and satisfy $\operatorname{Re} f_{\bar{z}} \geqslant\left|f_{z}\right|$ in the open disk and also that $f^{-1}(f(a))$ be countable for each $a$ in $D$. The second theorem requires that $f$ be a class $C^{1}$-function in a neighborhood of the disk satisfying $\left|f_{\bar{z}}\right|>\left|f_{z}\right|$ almost everywhere and $\operatorname{Re} f_{\bar{z}} \geqslant\left|f_{z}\right|$ everywhere inside the disk.
\end{abstract}

I. Introduction. This work contributes to the study of a certain basic question in the theory of uniform approximation in the complex plane, namely, to consider a continuous complex valued function defined on the closed unit disk in the complex plane and to analyze conditions which allow all continuous functions to be approximated uniformly on the disk by polynomials in the identity function and the given function. The question stems from the familiar and fundamental theorem of $\mathrm{K}$. Weierstrass which states that any continuous function on the disk can be approximated uniformly by polynomials in the identity function and its complex conjugate function. The ideas in this work have a debt to the paper of John Wermer [6] and they draw from the author's paper [5]. Further, the author owes much to many beneficial conversations with Andrew Browder about the problems dealt with here.

We denote by $\mathbf{C}$ the complex plane and by $z$ the identity function of $\mathbf{C}$ to $\mathbf{C}$ or its restrictions. Let $D$ be the set $\{s \in \mathbf{C}:|s| \leqslant 1\}$ and $C(D)$ be the set of all continuous complex valued functions on $D$. For $f$ in $C(D)$ we denote by $P_{f}$ the uniform closure on $D$ of all finite sums $\sum_{i, j \geqslant 0} a_{i j} z^{i} f^{j}$ where $a_{i j} \in \mathbf{C}$. We aim to present some progress in the understanding of when and why $P_{f}$ is $C(D)$.

Since $P_{f}$ always contains the constants, separates points, and is closed under uniform convergence, the Stone-Weierstrass theorem is available. However, it is not usual to prove $P_{f}=C(D)$ by showing that for each $g$ in $P_{f}$, the complex conjugate function $\bar{g}$ is also in $P_{f}$, except in very special cases, for example, when $f=a z+b \bar{z}$ where $a, b \in \mathbf{C}$ and $b \neq 0$. The usual methods of dealing with this problem come from two sources: functional analysis and the theory

Presented to the Society, September 4, 1974 under the title Another View of the Stone-Weierstrass Theorem; received by the editors November 7, 1974.

AMS (MOS) subject classifications (1970). Primary 46J10, 46J15; Secondary 30A36, 30A60, $30 \mathrm{~A} 82$.

1 This work was supported by The George C. Marshall Fund in Denmark while the author was visiting at Aarhus University. 
of several complex variables. In this work, we use only techniques of functional analysis and most of these can be termed classical. Some interesting applications of several complex variables to this setting may be found in [3].

Concerning the situation when $P_{f} \neq C(D)$, it is natural to seek a characterization of the elements of $P_{f}$. For instance, considering the case $f=|z|^{2}$, we see that $f$ is constant on any circle in $D$ with center at the origin, and so any function $g$ in $P_{f}$ is a uniform limit of polynomials in $z$ on each such circle. In this event, we know that $g$ must agree on each circle with a unique function which is continuous in the closed disk determined by the circle and analytic inside the circle. More generally, S. N. Mergelyan presents a characterization of $P_{f}$ for any real valued function $f$ in [4]. (Other results in this area may be found in [7].) Since we are limiting ourselves to the task of determining when $P_{f}=C(D)$, we would like to mention only the version of Mergelyan's result relevant to this, i.e., if $f$ in $C(D)$ is real valued, and if for each $a$ in $D$ the set $f^{-1}(f(a))$ has no interior and does not separate $\mathbf{C}$, then $P_{f}=C(D)$.

The paper of $J$. Wermer previously mentioned yields a result which plays an important guiding role for us. It states that if $f=\bar{z}+R$ where $R$ satisfies the Lipschitz condition $|R(s)-R(t)|<|s-t|$ for all $s, t$ in $D$ with $s \neq t$, then $P_{f}=C(D)$. Roughly speaking, it states that we can replace the function $\bar{z}$ in the Weierstrass theorem by a function which is "near" $\bar{z}$ and still retain the original conclusion. Concerning the term "near", it should be pointed out that there exist functions $f$ arbitrarily close to $\bar{z}$ in the uniform norm on $D$ for which $P_{f} \neq C(D)$; for example, approximate $\bar{z}$ by functions vanishing in a neighborhood of zero. Also if the function $f=\bar{z}+R$ is a smooth function and if $f$ is "near" $\bar{z}$ in a class $C^{1}$-sense, then $R$ easily satisfies the Lipschitz condition so that $f$ satisfies Wermer's theorem. However, in addition, this smooth case provides another link to the function $\bar{z}$. In fact, we shall show that when $R$ satisfies a slightly stronger Lipschitz condition, then $f$ is "like" $\bar{z}$ in the sense that, regarded as a map from $E_{2}$ to $E_{2}, f$ has negative Jacobian determinant everywhere. This last property may be expressed in terms of the differential operators $\partial / \partial z=\frac{1}{2}(\partial / \partial x-i \partial / \partial y), \partial / \partial \bar{z}=\frac{1}{2}(\partial / \partial x+i \partial / \partial y)$ applied to $f$ by (the inequality) $\left|f_{\bar{z}}\right|>\left|f_{z}\right|$ everywhere. And this fact leads to the reasonable conjecture that if $f$ is a smooth function in a neighborhood of $D$ such that $\left|f_{\bar{z}}\right|>\left|f_{z}\right|$ everywhere, then $P_{f}=C(D)$. We have not been able to prove the conjecture, but in [5] we proved the hypotheses of the conjecture imply that $C(D)$ is the uniform closure on $D$ of rational functions in $z$ and $f$ which are finite. And as a consequence of the present work, we verify the conjecture under the stronger condition $\operatorname{Re} f_{\bar{z}}>\left|f_{z}\right|$.

More specifically, we aim to present two settings which yield $P_{f}=C(D)$. In both theorems we will require the function $f$ to satisfy the condition $\operatorname{Re} f_{\bar{z}}$ $\geqslant\left|f_{z}\right|$ in the interior of the unit disk as well as some regularity condition. The first theorem is proved in $\S$ II and involves the notion of $f$ being differentiable at a point, say $z_{0}$, i.e.,

$$
f=f\left(z_{0}\right)+f_{x}\left(z_{0}\right)\left(x-x_{0}\right)+f_{y}\left(z_{0}\right)\left(y-y_{0}\right)+o\left(\left|z-z_{0}\right|\right) .
$$

THeOREM A. Let $f$ be in $C(D)$ and satisfy

(i) $f$ is differentiable in $\{s:|s|<1\}$,

(ii) $\operatorname{Re} f_{\bar{z}} \geqslant\left|f_{z}\right|$ everywhere in $\{s:|s|<1\}$, 
(iii) $f^{-1}(f(a))$ is countable for each a in D.

Then $P_{f}=C(D)$.

The second theorem concerns a smooth function and is proved in §III.

THEOREM B. Let $f$ be a class $C^{1}$-function in a neighborhood of $D$ such that $\left|f_{\bar{z}}\right|>\left|f_{z}\right|$ almost everywhere with $\operatorname{Re} f_{\bar{z}} \geqslant\left|f_{z}\right|$ everywhere in $\{s:|s|<1\}$. Then $P_{f}=C(D)$.

Finally, in $\S \mathrm{IV}$ we offer some closing remarks including an observation regarding approximation by $z$ and $f$ in an $L^{p}$-sense.

II. Proof of Theorem A. The main ideas in the proof can be termed "classical" in the best sense; $P_{f}$ is a closed subspace of $C(D)$, so by a standard corollary of the Hahn-Banach theorem, $P_{f}=C(D)$ if and only if there exists no nonzero continuous linear functional on $C(D)$ which annihilates $P_{f}$. According to the well-known representation theorem of F. Riesz (due in full generality to Kakutani), each continuous linear functional on $C(D)$ is of the form $g \rightarrow \int g d \mu$, where $\mu$ is a (uniquely determined) complex regular Borel measure on $D$. Thus, to show that $P_{f}=C(D)$, it suffices to show that if $\mu$ is a complex Borel measure on $D$ such that $\int g d \mu=0$ for all $g \in P_{f}$ (or more briefly, $\mu \perp P_{f}$ ), then $\mu$ is the zero measure. The argument then gets its usefulness from some important properties of compactly supported measures in $\mathbf{C}$ which we now quote in terms of our problem. The proofs may be found in [1].

Lebesgue two-dimensional measure in $\mathbf{C}$ will be denoted by $m$ and for any measure $\mu$ on $D,|\mu|$ will denote the associated positive total variation measure.

Definition. Let $\mu$ be a measure on $D$. For all $s \in \mathbf{C}$, we put $\tilde{\mu}(s)$ $=\int d|\mu| /|z-s|$.

LEMMA II.1. With $\mu$ as above, $\tilde{\mu}$ is integrable over any compact set (with respect to $m)$; in particular, $\tilde{\mu}<\infty$ a.e. $(m)$.

Definition. With $\mu$ as above, for each $s \in \mathbf{C}$ such that $\tilde{\mu}(s)<\infty$, we define $\hat{\mu}(s)=\int d \mu /(z-s)$.

Thus the function $\hat{\mu}$ is defined a.e. $(m)$ and integrable over bounded sets. A key role in this proof is played by

Lemma II.2. For any open set $U$ in $\mathbf{C}$ such that $\hat{\mu}(s)=0$ for almost all $s$ in $U$, we have $|\mu|(U)=0$; in particular, $\mu=0$ if $\hat{\mu}(s)=0$ a.e.

We can now continue with the proof. Let $\mu$ be a measure on $D$ such that $\mu \perp P_{f}$. We will show $\mu=0$. For any $a, a \notin D$, we have $(z-a)^{-1}$ $=-\sum_{0}^{\infty} z^{n} / a^{n+1}$, the series converging uniformly on $D$, and since $\int z^{n} d \mu=0$ for all $n$, we have $\hat{\mu}(a)=0$. By the last lemma, this reduces our problem of showing $\mu=0$ to the problem of showing $\hat{\mu}(s)=0$ for almost all $s$ in $D$.

Now put $E=\{b \in D: \mu(\{b\}) \neq 0\}$. $E$ is a countable set. For each $b \in E$, put $E_{b}=f^{-1}(f(b))$ and let $F=\cup_{b \in E} E_{b}$. By hypothesis the set $F$ has measure zero. Hence, to prove the theorem it suffices to take $a \in D-F$ with $\tilde{\mu}(a)<\infty$ and to show $\hat{\mu}(a)=0$. Let $a$ be such a point and put $\lambda$ $=(z-a)(f-f(a))$. We claim that $\lambda$ maps $D$ into $\{s \in \mathbf{C}: \operatorname{Re} s \geqslant 0\}$. Let us temporarily assume the claim and finish the proof of the theorem. For 
$n=1,2, \ldots$, put $p_{n}=\left(z+n^{-1}\right)^{-1}$ and $g_{n}=(f-f(a)) p_{n} \circ \lambda$. Since the image of $D$ under $\lambda$ is a compact subset of the right half-plane, each $p_{n}$ is uniformly approximable on that image by polynomials (in fact, it equals a power series expansion). It follows straightforwardly that $g_{n} \in P_{f}$ for each $n$ and also that $g_{n} \rightarrow(f-f(a)) /(f-f(a))(z-a)$ in $D$ so that $g_{n} \rightarrow(z-a)^{-1}$ in $D-f^{-1}(f(a))$. But $f^{-1}(f(a))$ is a countable set by hypothesis and is disjoint from $E$ by our choice of $a$ and hence is a set of $\mu$-measure zero. In addition, since $\left|p_{n}\right| \leqslant|z|^{-1}$ in the right half-plane (independent of $n$ ) we have $\left|g_{n}\right| \leqslant|z-a|^{-1}$ in $D$ and (by dominated convergence) $\hat{\mu}(a)=\lim \int g_{n} d \mu$ $=0$. Thus, Theorem $\mathrm{A}$ is proved once we have established that

$$
\operatorname{Re}[(t-s)(f(t)-f(s))] \geqslant 0
$$

whenever $s, t \in D$ and this is what we show now. Fix $s, t$ in $D$ and put $F=\operatorname{Re}[(t-s) f]$ so that $\operatorname{Re}[(t-s)(f(t)-f(s))]=F(t)-F(s)$. Our hypothesis on $f=u+i v$ is enough to conclude that $F(t)-F(s)=d F_{z_{0}}(t-s)$ where $z_{0}$ sits on the open line segment joining $s$ to $t$ (it is simply the Mean Value Theorem of elementary calculus applied to this situation). Furthermore, the following equalities are true since $F=\operatorname{Re}(t-s) u-\operatorname{Im}(t-s) v$;

$$
\begin{aligned}
d F(t-s) & =\operatorname{Re}(t-s) d u(t-s)-\operatorname{Im}(t-s) d v(t-s) \\
& =\operatorname{Re}\left[(t-s)\left((t-s) f_{z}+(\bar{t}-\bar{s}) f_{\bar{z}}\right)\right] .
\end{aligned}
$$

However, we have assumed that $\operatorname{Re} f_{\bar{z}} \geqslant\left|f_{z}\right|$ everywhere in $\{s:|s|<1\}$, so that this last expression is nonnegative. We conclude $d F_{z_{0}}(t-s) \geqslant 0$ which says exactly that $\operatorname{Re}[(t-s)(f(t)-f(s))] \geqslant 0$ and Theorem $\mathrm{A}$ is proved.

III. Proof of Theorem B. The main ideas in the proof are the same as those in the previous situation except that the smoothness condition on $f$ relaxes somewhat the problem of showing $\hat{\mu}(a)=0$ for almost all $a$ in $D$. The fact is that because of the lemma which follows, that problem is reduced to the case when $\mu$ is absolutely continuous with respect to two-dimensional Lebesgue measure.

LEMMA III.1. For any measure $\mu$ on $D$ and any function $g$ of class $C^{1}$ having compact support in $\mathbf{C}$, we have $\int_{D} g d \mu=\pi^{-1} \int_{\mathbf{C}} g_{\bar{z}} \hat{\mu} d m$.

(The proof may be found in [1].)

Let $\mu$ be a measure on $D$ such that $\mu \perp P_{f}$. We show $\mu=0$. Exactly as before, this reduces to showing $\hat{\mu}(s)=0$ for almost all $s$ in $D$. Suppose first that $\mu$ is absolutely continuous with respect to two-dimensional Lebesgue measure. Fix any $a$ in $D$ with $\tilde{\mu}(a)<\infty$ and again put $g_{n}=(f-f(a)) p_{n} \circ \lambda$ where $\lambda=(z-a)(f-f(a))$ and $p_{n}=\left(z+n^{-1}\right)^{-1}$. In the same way as before we have $g_{n} \in P_{f},\left|g_{n}\right| \leqslant|z-a|^{-1}$ in $D$ and $g_{n} \rightarrow(z-a)^{-1}$ in $D$ $-f^{-1}(f(a))$. And we could conclude $\hat{\mu}(a)=0$ (by dominated convergence) provided that $f^{-1}(f(a))$ has Lebesgue measure zero (and hence, $\mu$-measure zero). But this follows readily from the fact that $\left|f_{\bar{z}}\right|>\left|f_{z}\right|$ a.e. because the Jacobian determinant for $f$ is equal to $\left|f_{z}\right|^{2}-\left|f_{\bar{z}}\right|^{2}$ which is negative almost everywhere, while at the same time we must have $\left.\int_{f^{-1}(f(a))}|| f_{z}\right|^{2}-\left|f_{\bar{z}}\right|^{2} \mid d m$ $=0$. This last fact can be determined by examining the identity 


$$
\left.\int_{f^{-1}(f(a))}|| f_{z}\right|^{2}-\left|f_{\bar{z}}\right|^{2} \mid d m=\int_{\mathbf{C}} \#\left\{f^{-1}(f(a)) \cap f^{-1}(w)\right\} d m(w) .
$$

The identity may be found in [2] where it is shown that the function defined in all $\mathbf{C}$ by $F(w)=\#\left\{A \cap f^{-1}(w)\right\}=$ the number of elements in the set $A \cap f^{-1}(w)$ is Lebesgue measurable for any measurable set $A$. In our context, of course, $A=f^{-1}(f(a))$ so that $F(w)$ is identically zero except for one point, namely, $w=f(a)$, and so the integral of $F$ vanishes. Finally, let $\mu$ be an arbitrary measure which annhilates $P_{f}$. By the previous lemma we know $\int g d \mu=\pi^{-1} \int g_{\bar{z}} \hat{\mu} d m$ for any $C^{1}$-function $g$. We deduce that for any nonnegative integers $k, l$

$$
\int z^{k} f^{l} f_{\bar{z}} \hat{\mu} d m=\pi \int z^{k} \frac{f^{l+1}}{l+1} d \mu=0 .
$$

Thus $f_{\bar{z}} \hat{\mu} m$ is an annihilating measure for $P_{f}$, absolutely continuous with respect to $m$. By what we proved above, we have $f_{\bar{z}} \hat{\mu}=0$ a.e. $(m)$. Since $f_{\bar{z}} \neq 0$ a.e., we conclude $\hat{\mu}=0$ a.e., and hence that $\mu=0$. The theorem follows.

IV. Remarks. 1. The proof of Theorem A still holds up if the hypothesis on $f$ is weakened from " $f$ differentiable" to " $f$ Gateaux differentiable".

2. The part of the proof of Theorem B which shows that any annihilating measure for $P_{f}$ which is absolutely continuous with respect to two-dimensional Lebesgue measure is the zero measure still holds up if the hypothesis on $f$ in Theorem $\mathrm{B}$ is weakened to $f$ being differentiable or even to $f$ being Gateaux differentiable. Hence, in that event, there are no such absolutely continuous annihilating measures and, for example, we get $L^{p}(\mathrm{dm})$ density of polynomials in $z$ and $f$; in fact, we get weak-star density in $L^{\infty}(\mathrm{dm})$.

3. Concerning the function $f=\bar{z}+R$ of Wermer's paper [6], we cannot conclude that $\left|f_{\bar{z}}\right|>\left|f_{z}\right|$ everywhere, never mind the stronger fact $\operatorname{Re} f_{\bar{z}}$ $>\left|f_{z}\right|$ (for instance, if $\left.R=z \bar{z} / 4+\bar{z}^{2} / 4\right)$. However, if $R$ satisfies $|R(t)-R(s)|$ $\leqslant k|t-s|$ for some constant $k$ with $0<k<1$, then we do get $\operatorname{Re} f_{\bar{z}}>\left|f_{z}\right|$. For it is not hard to show that $\left|R_{\bar{z}}\right|+\left|R_{z}\right| \leqslant k$ and it is clear that $f_{z}=R_{z}$, $\operatorname{Re} f_{\bar{z}}=1+\operatorname{Re} R_{\bar{z}}$, so that $\operatorname{Re} f_{\bar{z}} \geqslant 1-\left|R_{\bar{z}}\right|>\left|R_{z}\right|$. Thus, Wermer's theorem (in weak form) follows from Theorem A.

\section{REFERENCES}

1. A. Browder, Introduction to function algebras, Benjamin, New York, 1969. MR 39 \# 7431.

2. H. Federer, Geometric measure theory, Die Grundlehren der math. Wissenschaften, Band 153, Springer-Verlag, New York, 1969. MR 41 \# 1976.

3. L. Hörmander and J. Wermer, Uniform approximation on compact sets in $C^{n}$, Math. Scand. 23 (1968), 5-21 (1969). MR 40 \# 7484.

4. S. N. Mergeljan, On the representation of functions by series of polynomials on closed sets, Dokl. Akad. Nauk SSSR 78 (1951), 405-408; English transl., Amer. Math. Soc. Transl. (1) 13 (1962), 287-293. MR 13, 23.

5. K. J. Preskenis, Approximation on disks, Trans. Amer. Math. Soc. 171 (1972), 445-467. MR $47 \# 685$.

6. J. Wermer, Approximation on a disk, Math. Ann. 155 (1964), 331-333. MR 29 \#2670.

7. - Polynomially convex disks, Math. Ann. 158 (1965), 6-10. MR 30 \#158.

Department of Mathematics, Aarhus University, Aarhus, Denmark

Current address: Department of Mathematics, Boston College, Chesnut Hill, Massachusetts 02167 